University of Nebraska - Lincoln

DigitalCommons@University of Nebraska - Lincoln

Faculty Publications from Nebraska Center for

Materials and Nanoscience, Nebraska Center Materials and Nanoscience

for (NCMN)

February 2005

\title{
Wave attenuations in solids with perfectly aligned cracks
}

Liyong Yang

University of Nebraska - Lincoln, lyang4@unl.edu

Joseph A. Turner

University of Nebraska - Lincoln, jaturner@unl.edu

Follow this and additional works at: https://digitalcommons.unl.edu/cmrafacpub

Part of the Nanoscience and Nanotechnology Commons

Yang, Liyong and Turner, Joseph A., "Wave attenuations in solids with perfectly aligned cracks" (2005). Faculty Publications from Nebraska Center for Materials and Nanoscience. 53.

https://digitalcommons.unl.edu/cmrafacpub/53

This Article is brought to you for free and open access by the Materials and Nanoscience, Nebraska Center for (NCMN) at DigitalCommons@University of Nebraska - Lincoln. It has been accepted for inclusion in Faculty Publications from Nebraska Center for Materials and Nanoscience by an authorized administrator of DigitalCommons@University of Nebraska - Lincoln. 


\title{
Wave attenuations in solids with perfectly aligned cracks
}

\author{
Liyong Yanga) and Joseph A. Turner \\ Department of Engineering Mechanics, Center for Material Research and Analysis, W317.4 Nebraska Hall, \\ University of Nebraska-Lincoln, Lincoln, Nebraska 68588-0526
}

\begin{abstract}
The theory of wave propagation and scattering in cracked media is applied to study the wave attenuations in an isotropic solid medium containing perfectly aligned penny-shaped microcracks. The unit normals of all cracks are assumed parallel to a given direction. The wave scattering model is formulated using an anisotropic Green's dyadic approach. Explicit expressions are derived for attenuations of the three wave modes in terms of the microcrack density. Numerical results are presented and discussed. In particular, comparisons of the attenuation results presented in this letter with previous results for the Rayleigh limit are given.

(C) 2005 Acoustical Society of America

PACS numbers: 43.20Bi, 43.20Gp, 43.35Cg [AN]

Date Received: 8 September $2004 \quad$ Date Accepted: 16 February 2005
\end{abstract}

\section{Introduction}

The study of elastic wave scattering from cracks is important for various areas of engineering and geophysics, in particular in ultrasonic nondestructive evaluation and materials characterization. Quantitative assessment of damage using nondestructive methods is essential for determining the structural integrity of structures and for predicting the remaining usable life. Changes in the material response due to the reduction of effective elastic stiffness resulting from microcrack damage have a significant influence on the physical properties of the materials. Distributed microcracks often give rise to a decrease in wave velocity and an increase in attenuation. Precise knowledge of the attenuation and wave velocity of ultrasonic waves in cracked media provides a direct approach for quantifying the material damage. Several recent studies associated with wave propagation in cracked media have been reviewed in the literature [Yang and Turner, 2003; Eriksson et al., 1995; Zhang and Gross, 1993]. In a previous paper [Yang and Turner, 2003], a theory was developed to describe the scattering of elastic waves by uniaxially aligned penny-shaped cracks. Such cracks often result from uniaxial compressive loading. However, in the case of structural materials subjected to the uniaxial tension, the status of damage is generally defined as that of perfectly aligned cracks. In this case, the unit normals of all cracks are perpendicular to the plane of isotropy as studied by Hudson (1981). His investigations of wave attenuation in cracked solids were restricted to the Rayleigh regime. Outside the Rayleigh limit, examinations of the attenuation have not been examined for this situation. In this letter, the attenuation of elastic waves from perfectly aligned cracks is studied. Explicit expressions of attenuations of the shear horizontal ( $\mathrm{SH}$ ), quasilongitudinal (qP), and quasishear vertical (qSV) waves are given. In the Rayleigh limit, comparisons of numerical results obtained in this letter and those presented by Hudson (1981) are particularly addressed.

\section{Effective properties}

For perfectly aligned cracks, the unit normals of all microcracks are perpendicular to the plane of isotropy. As such, the overall properties of the cracked solid are transversely isotropic with symmetry axis in the $x_{3}$ axis ( $\hat{\mathbf{n}}$ direction). Therefore, the microcrack density distribution is given by

$$
\zeta(\hat{\mathbf{n}}, \hat{\mathbf{m}})=2 \pi[\delta(\hat{\mathbf{n}}-\hat{\mathbf{m}})+\delta(\hat{\mathbf{n}}+\hat{\mathbf{m}})]
$$

a)Electronic mail: lyang@bigred.unl.edu 


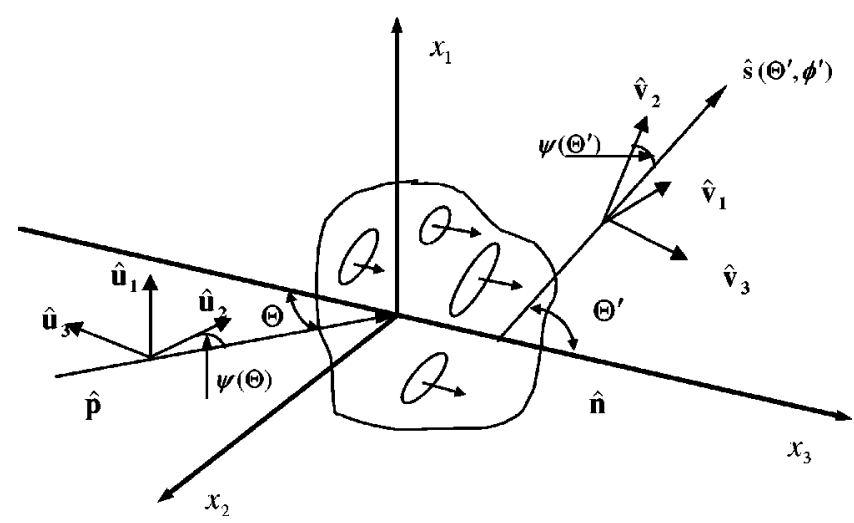

Fig. 1. Geometry for the propagation direction $\hat{\mathbf{p}}$, the scattered direction $\hat{\mathbf{s}}$, and the respective polarization direction $\hat{\mathbf{u}}$ and $\hat{\mathbf{v}}$ in the local coordinate system.

where the unit vector $\hat{\mathbf{m}}$ is the unit normal of each crack. The effective stiffness caused by all microcracks per unit volume is weighted by the crack density function over the single-crack Green's function $C_{i j k l}^{(s)}$ given by $C_{i j k l}^{*}(\hat{\mathbf{n}})=(\varepsilon / 4 \pi) \int_{\Omega} C_{i j k l}^{(s)}(\hat{\mathbf{m}}) \zeta(\hat{\mathbf{n}}, \hat{\mathbf{m}}) d \Omega$ [Yang and Turner, 2003]. Then, the effective stiffness due to the distribution of perfectly aligned penny-shaped cracks is derived as

$$
\mathbf{C}^{*}=\varepsilon b_{i} \mathbf{I}^{i},
$$

where the coefficients $b_{i}$ are

$$
\begin{gathered}
b_{1}=0, \quad b_{2}=2 \mu \frac{16 \nu^{2}(1-\nu)}{3(1-2 \nu)^{2}}, \quad b_{3}=b_{4}=2 \mu \frac{16 \nu(1-\nu)}{3(1-2 \nu)}, \\
b_{5}=2 \mu \frac{32(1-\nu)}{3(2-\nu)}, \quad b_{6}=-2 \mu \frac{16 \nu(1-\nu)}{3(2-\nu)} .
\end{gathered}
$$

The nondimensional microcrack density $\varepsilon$ is defined by $\varepsilon=N\left\langle a^{3}\right\rangle=\int_{a^{-}}^{a^{+}} A(a) a^{3} d a$, where $N$ is the number of microcracks of per unit volume. The tensors $\mathbf{I}^{i}$ are defined in terms of the unit vector $\hat{\mathbf{m}}$ and Kronecker delta function. The ensemble average stiffness may be represented by $\overline{\mathbf{C}}(\mathbf{x})$ $=\overline{\mathbf{C}}^{0}+\delta \overline{\mathbf{C}}(\mathbf{x})$, where $\overline{\mathbf{C}}^{0}=\langle\overline{\mathbf{C}}(\mathbf{x})\rangle=\mathbf{C}^{0}-\mathbf{C}^{*}$ is the ensemble average modulus tensor. Here, $\mathbf{C}^{0}$ is the stiffness tensor in the uncracked state.

\section{Attenuation}

The attenuations are calculated using stochastic operator theory with details given elsewhere [Yang and Turner, 2003]. The ensemble average material response is governed by the Dyson equation, which is easily solved for the effective wave number under the assumption of statistical homogeneity. The imaginary part of the wave number, a quantity proportional to the scattering attenuation, is a convolution between the material response without scattering and the covariance of the elastic moduli fluctuations. For a small concentration of microcracks, the covariance is written as $\left\langle\delta C_{i j k l}(\mathbf{x}) \delta C_{\alpha \beta \gamma \delta}(\mathbf{y})\right\rangle=\varepsilon \eta(r) \Xi_{i j k l}^{\alpha \beta \gamma \delta}$ with $r=|\mathbf{x}-\mathbf{y}|$. The expressions for attenuation require inner products on $\Xi$ given in terms of four unit vectors by $\Xi_{i j k l}^{\alpha \beta \gamma \delta} \hat{u}_{\beta} \hat{u}_{k} \hat{p}_{\alpha} \hat{p}_{l} \hat{s}_{i} \hat{s}_{\delta} \hat{v}_{\gamma} \hat{v}_{j}$. The vectors $\hat{\mathbf{p}}$ and $\hat{\mathbf{s}}$, respectively, represent the incoming and outgoing propagation directions and the vectors $\hat{\mathbf{u}}$ and $\hat{\mathbf{v}}$ are vectors defining the polarization directions of those waves.

Without loss of generality, a reference plane is defined as the $\hat{\mathbf{p}}-\hat{\mathbf{n}}$ plane (see Fig. 1). Thus, the vectors $\hat{\mathbf{n}}, \hat{\mathbf{p}}$, and $\hat{\mathbf{s}}$ are defined with respect to a general $x_{1} x_{2} x_{3}$ coordinate system as 
$\hat{\mathbf{n}}=\hat{\mathbf{x}}_{3}, \quad \hat{\mathbf{p}}=\hat{\mathbf{x}}_{2} \sin \Theta+\hat{\mathbf{x}}_{3} \cos \Theta, \quad$ and $\quad \hat{\mathbf{s}}=\hat{\mathbf{x}}_{1} \sin \Theta^{\prime} \cos \phi^{\prime}+\hat{\mathbf{x}}_{2} \sin \Theta^{\prime} \sin \phi^{\prime}+\hat{\mathbf{x}}_{3} \cos \Theta^{\prime} . \quad$ The polarization vectors $\hat{\mathbf{u}}$ and $\hat{\mathbf{v}}$ are defined with respect to these angles and $\psi$ as $\hat{\mathbf{u}}_{1}=\hat{\mathbf{x}}_{1}, \hat{\mathbf{u}}_{2}$ $=\hat{\mathbf{x}}_{2} \sin \gamma+\hat{\mathbf{x}}_{3} \cos \gamma, \hat{\mathbf{u}}_{3}=-\hat{\mathbf{x}}_{2} \cos \gamma+\hat{\mathbf{x}}_{3} \sin \gamma$, and $\hat{\mathbf{v}}_{1}=\hat{\mathbf{x}}_{1} \sin \phi^{\prime}-\hat{\mathbf{x}}_{2} \cos \phi^{\prime}, \hat{\mathbf{v}}_{2}=\hat{\mathbf{x}}_{1} \sin \gamma^{\prime} \cos \phi^{\prime}$ $+\hat{\mathbf{x}}_{2} \sin \gamma^{\prime} \sin \phi^{\prime}+\hat{\mathbf{x}}_{3} \cos \gamma^{\prime}, \quad \hat{\mathbf{v}}_{3}=-\hat{\mathbf{x}}_{1} \cos \gamma^{\prime} \cos \phi^{\prime}-\hat{\mathbf{x}}_{2} \cos \gamma^{\prime} \sin \phi^{\prime}+\hat{\mathbf{x}}_{3} \sin \gamma^{\prime}$, where the angles $\gamma$ and $\gamma^{\prime}$ are defined by $\gamma=\Theta+\psi(\Theta)$ and $\gamma^{\prime}=\Theta^{\prime}+\psi\left(\Theta^{\prime}\right)$. Then, the required inner products are: for $\alpha_{\mathrm{SH}}$,

$$
\begin{aligned}
& \underset{\Xi \ldots \ldots}{\ldots \ldots \hat{\mathbf{u}} \hat{\mathbf{p}} \hat{\mathbf{p}} \hat{\mathbf{p}} \hat{\mathbf{v}}_{1} \mathbf{\mathbf { s }} \mathbf{v}_{1}}=\frac{b_{5}^{2}}{16} \cos ^{2} \Theta \cos ^{2} \Theta^{\prime} \sin ^{2} \phi^{\prime}, \\
& \Xi_{\ldots \ldots}^{\ldots \ldots \hat{\mathbf{u}} \hat{\mathbf{p}} \hat{\mathbf{p}} \hat{\mathbf{p}} \hat{\mathbf{p}} \hat{\mathbf{s}}_{2} \mathbf{v}_{2}}=\frac{b_{5}^{2}}{16} \cos ^{2} \Theta \sin ^{2}\left(\Theta^{\prime}+\gamma^{\prime}\right) \cos ^{2} \phi^{\prime}, \\
& \underset{\exists \ldots \ldots}{\ldots \ldots \hat{\mathbf{u}} \hat{\mathbf{p}} \hat{\mathbf{s}} \hat{\mathbf{v}}_{3}}=\frac{b_{5}^{2}}{16} \cos ^{2} \Theta \cos ^{2}\left(\Theta^{\prime}+\gamma^{\prime}\right) \cos ^{2} \phi^{\prime} ;
\end{aligned}
$$

for $\alpha_{\mathrm{qP}}$,

$$
\underset{\Xi_{1} \ldots \hat{\mathbf{u}}_{2} \hat{\mathbf{p}} \hat{\mathbf{p}} \hat{\mathbf{s}} \hat{\mathbf{v}}_{1}}{\mathbf{v}_{1}}=\frac{b_{5}^{2}}{16} \sin ^{2}(\Theta+\gamma) \cos ^{2} \Theta^{\prime} \cos ^{2} \phi^{\prime}
$$

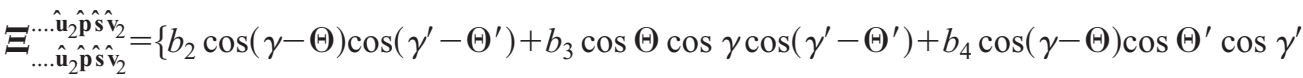

$$
\left.+\left(b_{5} / 4\right) \sin (\gamma+\Theta) \sin \left(\gamma^{\prime}+\Theta^{\prime}\right) \sin \phi^{\prime}+\left(b_{5}+b_{6}\right) \cos \Theta \cos \gamma \cos \Theta^{\prime} \cos \gamma^{\prime}\right\}^{2},
$$

$$
\Xi_{\ldots \ldots}^{\ldots} \hat{\mathbf{u}}_{2} \hat{\mathbf{p}} \hat{\mathbf{s}} \hat{\mathbf{v}}_{3} \hat{\hat{v}_{3}}=\left\{b_{2} \cos (\gamma-\Theta) \sin \left(\gamma^{\prime}-\Theta^{\prime}\right)+b_{3} \cos \Theta \cos \gamma \sin \left(\gamma^{\prime}-\Theta^{\prime}\right)+b_{4} \cos (\gamma-\Theta) \cos \Theta^{\prime} \sin \gamma^{\prime}\right.
$$$$
\left.-\left(b_{5} / 4\right) \sin (\gamma+\Theta) \cos \left(\gamma^{\prime}+\Theta^{\prime}\right) \sin \phi^{\prime}+\left(b_{5}+b_{6}\right) \cos \Theta \cos \gamma \cos \Theta^{\prime} \sin \gamma^{\prime}\right\}^{2} ;
$$

and, for $\alpha_{\mathrm{qSV}}$,

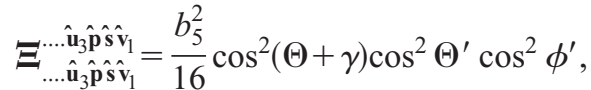

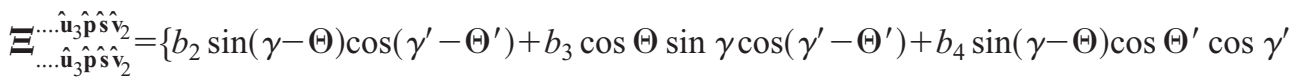

$$
\begin{aligned}
& \left.-\left(b_{5} / 4\right) \cos (\gamma+\Theta) \sin \left(\gamma^{\prime}+\Theta^{\prime}\right) \sin \phi^{\prime}+\left(b_{5}+b_{6}\right) \cos \Theta \sin \gamma \cos \Theta^{\prime} \cos \gamma^{\prime}\right\}^{2}, \\
& \Xi_{\ldots}^{\ldots . . . \hat{\mathbf{u}}_{3} \hat{\mathbf{p}} \hat{\mathbf{p}} \hat{\mathbf{v}}_{3} \hat{\mathbf{s}}_{3}}=\left\{b_{2} \sin (\gamma-\Theta) \sin \left(\gamma^{\prime}-\Theta^{\prime}\right)+b_{3} \cos \Theta \sin \gamma \sin \left(\gamma^{\prime}-\Theta^{\prime}\right)+b_{4} \sin (\gamma-\Theta) \cos \Theta^{\prime} \sin \gamma^{\prime}\right. \\
& \left.+\left(b_{5} / 4\right) \cos (\gamma+\Theta) \cos \left(\gamma^{\prime}+\Theta^{\prime}\right) \sin \phi^{\prime}+\left(b_{5}+b_{6}\right) \cos \Theta \sin \gamma \cos \Theta^{\prime} \sin \gamma^{\prime}\right\}^{2} .
\end{aligned}
$$

Here, the spatial correlation function $\eta$ is assumed to have a simple exponential form $\eta(r)=e^{-r / L}$, where $L$ is the correlation length, $L=2\langle a\rangle$. Thus, the resulting dimensionless attenuations are finally given in the form [Turner, 1999; Yang and Turner, 2003]

$$
\begin{aligned}
& \alpha_{\beta}(\hat{\mathbf{p}}) L=\frac{x_{\beta}^{4} C_{\beta}(\hat{\mathbf{p}}) \varepsilon}{2 \rho^{2}} \times\left\{\int \frac{\Xi_{\ldots \hat{\mathbf{u}}_{K} \hat{\mathbf{s}} \hat{\mathbf{v}}_{1}}^{\cdots} \hat{\mathbf{u}}_{K} \hat{\mathbf{p}} \hat{\mathbf{v}}_{1}}{\left(1+x_{\beta}^{2}(\hat{\mathbf{p}})+x_{\mathrm{SH}}^{2}(\hat{\mathbf{s}})-2 x_{\beta}(\hat{\mathbf{p}}) x_{\mathrm{SH}}(\hat{\mathbf{s}}) \hat{\mathbf{p}} \cdot \hat{\mathbf{s}}\right)^{2} C_{\mathrm{SH}}^{5}(\hat{\mathbf{s}})} d^{2} \hat{s}\right. \\
& +\int \frac{\Xi_{\ldots \hat{\mathbf{u}}_{K} \hat{\mathbf{p}} \hat{\mathbf{s}} \hat{\mathbf{v}}_{2}} \hat{\hat{\mathbf{u}}_{K} \hat{\mathbf{p}} \hat{\mathbf{s}} \hat{\mathbf{v}}_{2}}}{\left(1+x_{\beta}^{2}(\hat{\mathbf{p}})+x_{\mathrm{qP}}^{2}(\hat{\mathbf{s}})-2 x_{\beta}(\hat{\mathbf{p}}) x_{\mathrm{qP}}(\hat{\mathbf{s}}) \hat{\mathbf{p}} \cdot \hat{\mathbf{s}}\right)^{2} C_{\mathrm{qP}}^{5}(\hat{\mathbf{s}})} d^{2} \hat{s} \\
& \left.+\int \frac{\Xi_{\ldots, \hat{u}_{K} \hat{\mathbf{p}} \hat{\mathbf{s}} \hat{v}_{3}}}{\left(1+x_{\beta}^{2}(\hat{\mathbf{p}})+x_{\mathrm{qSV}}^{2}(\hat{\mathbf{s}})-2 x_{\beta}(\hat{\mathbf{p}}) x_{\mathrm{qSV}}(\hat{\mathbf{s}}) \hat{\mathbf{p}} \cdot \hat{\mathbf{s}}\right)^{2} C_{\mathrm{qSV}}^{5}(\hat{\mathbf{s}})} d^{2} \hat{s}\right\},
\end{aligned}
$$




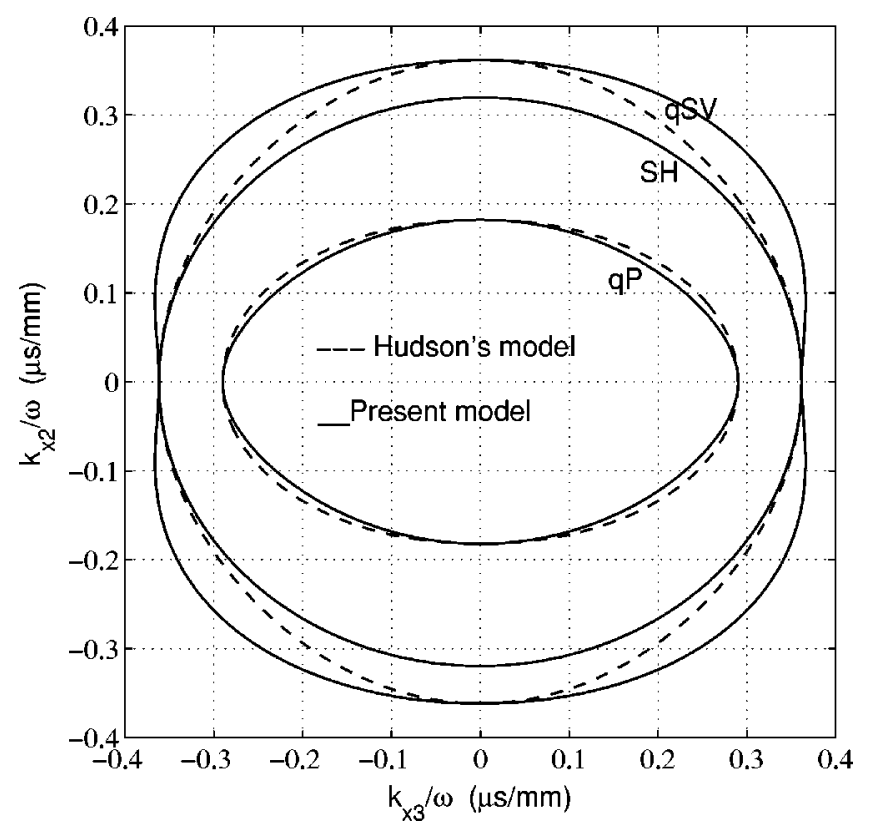

Fig. 2. Slowness surfaces for damage density $\varepsilon=0.1$.

where $K$ is defined as the polarization for the wave type $\beta$. In the long wavelength Rayleigh limit, $x_{\beta} \ll 1$, Eq. (7) can be simplified to

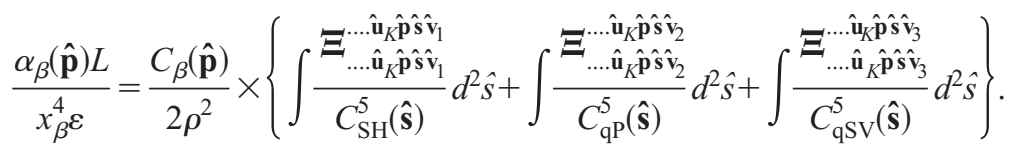

\section{Results}

Numerical results are now presented for a specific case, in which the observed transverse isotropy of the cracked material is entirely due to the presence of the perfectly aligned cracks. The material properties of the uncracked material used are Young's modulus $E=2.0 \times 10^{11} \mathrm{~Pa}$, Poisson's ratio $\nu=0.3$, and density $\rho=7860 \mathrm{~kg} / \mathrm{m}^{3}$. First, the slowness surfaces are presented for damage density $\varepsilon=0.1$ as shown in Fig. 2. Exact agreement of the results for SH waves between the present model and Hudson's model is seen in Fig. 2. However, the slowness curves of the qP and qSV waves have a more limited range of agreement. This discrepancy is thought to be due to the assumption of nonvarying polarization in the work of Hudson.

The attenuations within the Rayleigh limit are calculated using Eq. (8). The angular dependence of three attenuations is described by the quantity $\alpha_{\beta} L /\left(x_{\beta}^{4} \varepsilon\right)$. Those parameters for each wave type are shown in Figs. 3, 4, and 5, respectively. The attenuation for SH waves for damage density at $\varepsilon=0.01$ and $\varepsilon=0.05$ is presented first in Fig. 3. In order to compare the present results with those studied by Hudson (1981), the equivalent parameter is also shown in Fig. 3 (dashed line). It is seen that for two different damage densities, the attenuations of Hudson's model are identical. This result is due to the fact that Hudson uses the velocities of the uncracked solid to calculate the attenuation, rather than the velocities of the cracked solid as used here.

In Fig. 4, the Rayleigh attenuations of the $\mathrm{qP}$ wave are presented for both the present model and Hudson's model. The difference between the two models is attributed to the same reasons as discussed earlier for SH waves. The Rayleigh attenuations of the $\mathrm{qSV}$ wave are shown in Fig. 5. It is seen from Fig. 5 that the comparison of Rayleigh attenuation for the qSV waves between the present model and Hudson's model is significant for damage density $\varepsilon=0.05$ near 


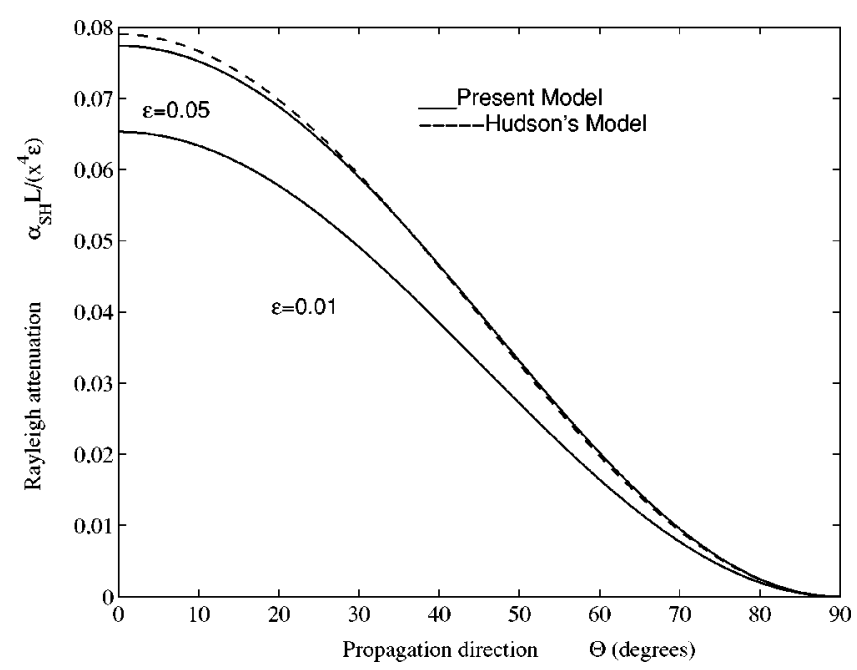

Fig. 3. Rayleigh attenuation, $\alpha_{\mathrm{SH}} L /\left(x^{4} \varepsilon\right)$ as a function of direction.

$\Theta=45^{\circ}$. This difference occurs in a region for which the wave speed in the cracked solid varies dramatically from the background medium. Since the attenuations are dependent on combinations of eight wave vectors, a small deviation in wave speed can have a pronounced effect.

The results outside the Rayleigh regime are calculated using the complete integrals, Eq. (7). The directional dependence of the attenuations is presented in terms of the single dimensionless frequency $x_{\mathrm{SH}}=1.0$ in Fig. 6 . The normalized SH, qP, and qSV attenuations are shown as a function of propagation direction at the damage density $\varepsilon=0.05$. The attenuations of the $\mathrm{qP}$ and $\mathrm{SH}$ waves for propagation parallel to the crack alignment direction are seen to be maximum. The direction of maximum qSV attenuation is in the vicinity of $\Theta=45^{\circ}$. In general, the direction of the peak of the qSV attenuation is dependent on both frequency and damage.

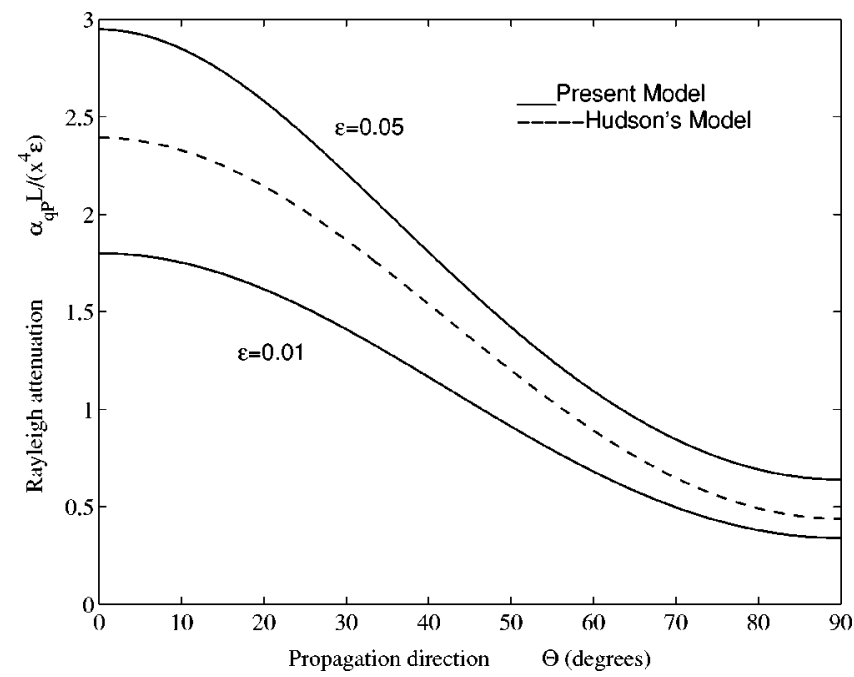

Fig. 4. Rayleigh attenuation, $\alpha_{\mathrm{qP}} L /\left(x^{4} \varepsilon\right)$ as a function of direction. 


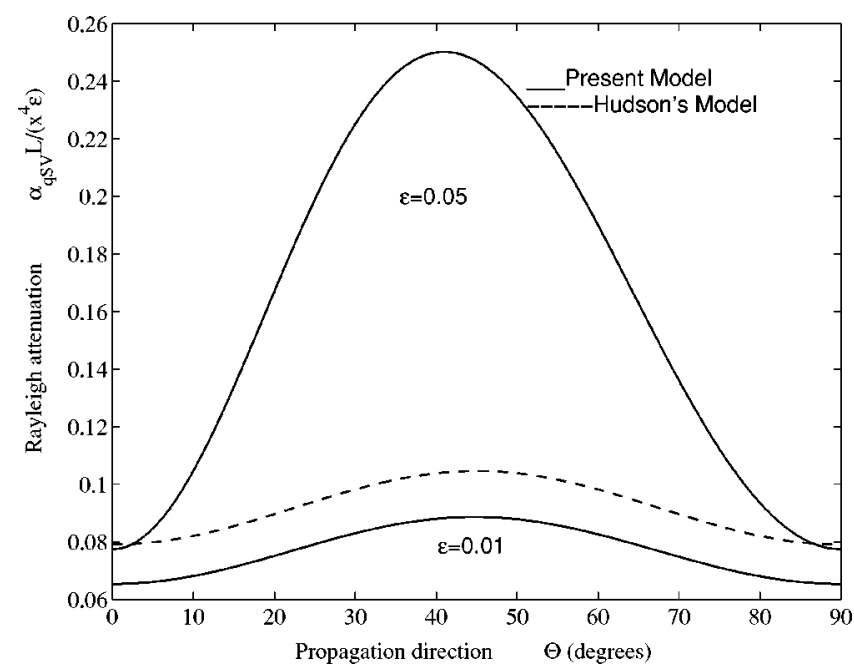

Fig. 5. Rayleigh attenuation, $\alpha_{\mathrm{qSV}} L /\left(x^{4} \varepsilon\right)$ as a function of direction.

It is observed that the maximum attenuation of $\mathrm{SH}$ wave is at $\Theta=0^{\circ}$, parallel to the normals of cracks, and at $\Theta=90^{\circ}$ there is no attenuation. As expected, the cracks cause maximum effects of interacting with wave motion when the surfaces of the cracks are perpendicular to the direction of wave propagation. It is interesting to note that even when the $\mathrm{qP}$ and $\mathrm{qSV}$ waves propagate perpendicular to the normals of the cracks $\left(\Theta=90^{\circ}\right)$, the attenuations are not zero. It is speculated that the attenuation arises from the Poisson effect which couples the qP and qSV waves at the crack. However, since the SH wave is polarized in the direction perpendicular to the crack normal, the cracks do not hinder the wave propagation.

Finally, in Fig. 7 the normalized SH, qP, and qSV attenuations are plotted versus dimensionless frequency, $x_{\mathrm{SH}}$, for a single propagation direction $\left(\Theta=60^{\circ}\right)$ for damage density $\varepsilon=0.05$. The frequency dependence of the attenuations is seen clearly. Also, the relative values of

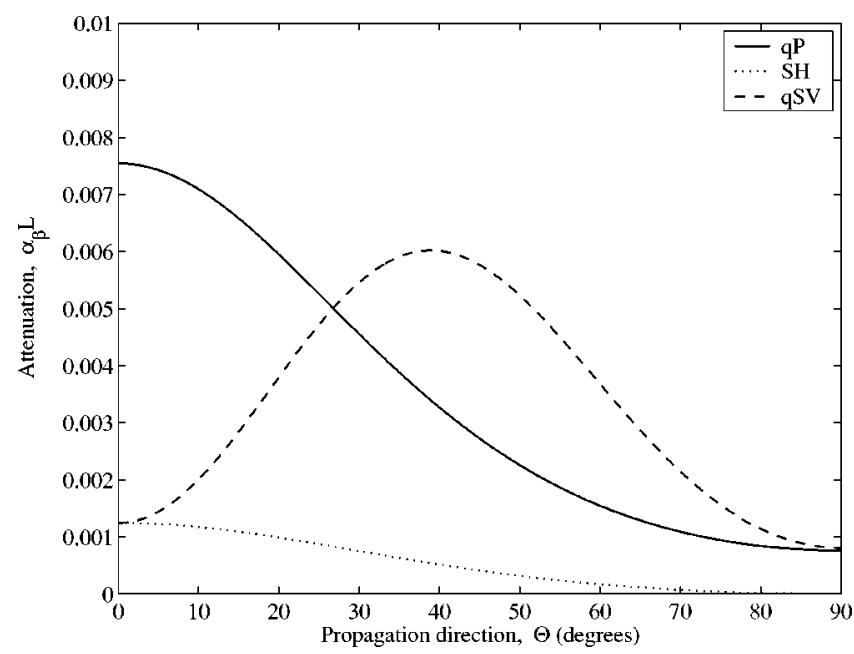

Fig. 6. Angular dependence of attenuations, $\alpha_{\beta} L$, for the damage density $\varepsilon=0.05$ at frequency $x_{\mathrm{SH}}=1.0$ 


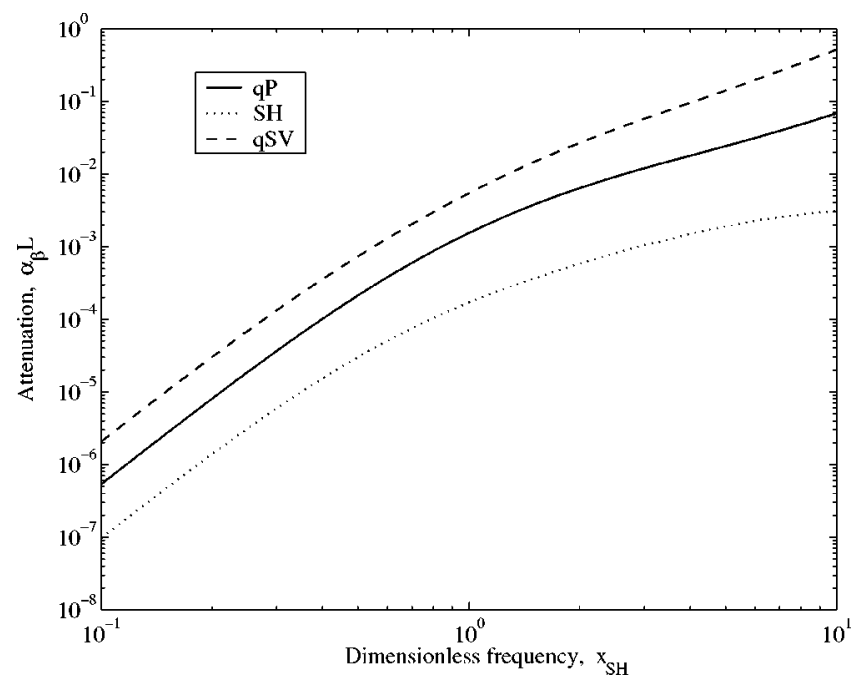

Fig. 7. Normalized attenuations, $\alpha_{\beta} L$, as a function of dimensionless frequency, $x_{\mathrm{SH}}$, for the propagation direction of $60^{\circ}$ at damage density $\varepsilon=0.05$.

the attenuations of each mode type are evident with ordering of $\alpha_{\mathrm{qSV}}>\alpha_{\mathrm{qP}}>\alpha_{\mathrm{SH}}$ for the entire region shown.

\section{Conclusions}

The attenuations of elastic waves in solids with perfectly aligned cracks have been examined and are shown to depend on frequency, phase velocity, propagation direction, and damage density as expected. Comparisons of the present model with Hudson's model in the Rayleigh limit show that the results presented here are more widely applicable than those presented by Hudson particularly for cases in which the crack-induced anisotropy is strong. The simple form of the results makes them particularly useful for nondestructive testing and evaluation.

\section{Acknowledgment}

The National Science Foundation (Grant Nos. CMS-9978707 and INT-0089548) is gratefully acknowledged for supporting this work.

Eriksson, A. S., Bostrom, A., and Datta, S. K. (1995). "Ultrasonic wave propagation through a cracked solid," Wave Motion 22, 297-310.

Hudson, J. A. (1981). “Wave speeds and attenuation of elastic waves in material containing cracks,” Geophys. J. R. Astron. Soc. 64, 133-150.

Turner, J. A. (1999). "Elastic wave propagation and scattering in heterogeneous, anisotropic media: Textured polycrystalline materials," J. Acoust. Soc. Am. 106, 541-552.

Yang, L., and Turner, J. A. (2003). "Elastic wave propagation and scattering in solids with uniaxially aligned cracks," J. Acoust. Soc. Am. 114, 591-600.

Zhang, C., and Gross, D. (1993). "Wave attenuation and dispersion in randomly cracked solids-II. Penny-shaped cracks,” Int. J. Eng. Sci. 31, 859-872. 